\title{
Gradient extraction operators for discrete interval-valued data
}

\author{
C. Lopez-Molina ${ }^{1,2}$ C. Marco-Detchart ${ }^{1}$ J. Cerron $^{1}$ H. Bustince ${ }^{1}$ B. De Baets ${ }^{2}$ \\ ${ }^{1}$ Dpto. Automatica y Computacion, Universidad Publica de Navarra, Pamplona (Spain) \\ ${ }^{2}$ KERMIT, Dept. of Mathematical Modelling, Statistics and Bioinformatics, Ghent University, Ghent (Belgium)
}

\begin{abstract}
Digital images are generally created as discrete measurements of light, as performed by dedicated sensors. Consequently, each pixel contains a discrete approximation of the light inciding in a sensor element. The nature of this measurement implies certain uncertainty due to discretization matters. In this work we propose to model such uncertainty using intervals, further leading to the generation of so-called interval-valued images. Then, we study the partial differentiation of such images, putting a spotlight on antisymmetric convolution operators for such task. Finally, we illustrate the utility of the interval-valued images by studying the behaviour of an extended version of the well-known Canny edges detection method.
\end{abstract}

Keywords: Image processing, Interval-valued information, Edge detection, Canny method

\section{Introduction}

Mainly due to economic matters, digital sampling has become the most common way to record and store continuous facts, being video or audio sampling paradigmatic examples of such. However, it is evident that discretization processes miss (or lose) a portion of the initial information, simply because the range of possible measurements is limited to a predefined set. In the case of a digital image, the loss of information due to the model (the limitation in the number of tones) is combined with that alien to it (as noise or broken cells in the sensor). As a consequence, we can never be fully certain about the tone of a pixel, this fact having a variable impact on different image processing tasks. In the field of edge detection the problems originating from this uncertainty manifest themselves clearly, since sometimes not even two humans can reach an agreement on where the boundary between two objects is.

Considering the inherent ambiguity of the information in a digital image, fuzzy logic, either in a narrow or wide sense [1], appears as an appropriate tool for low level feature extraction. In fact, within the context of image processing, edge detection has been one of the tasks for which fuzzy logic has been most prolific. It has been used for different purposes at almost any step of the process, from the very interpretation of the image $[2,3]$ to the reconstruction of the edges once they have been characterized [4]. Authors have experimented with a wide variety of techniques based on fuzzy logic, including fuzzy inference systems [4], fuzzy morphology [5] and fuzzy peer groups [6]. Many of such attempts are based on representing images as classical Fuzzy Sets (FS), for which the membership degree of each element is expressed in the interval $[0,1]$. However, apart from FSs, many extensions have been proposed in order to model fuzziness for edge detection. Among these extensions, some relevant examples Interval-Valued Fuzzy Sets (IVFSs), which represent membership degrees by means of intervals [7], or Type-2 Fuzzy Sets (T2FSs), which represent membership degrees as FSs [8].

In this work we study a novel interval-valued representation of images, which is constructed to capture the inherent ambiguity in the image capturing (and discretization) process. Then, we analyze the computation of gradients on such representation, what leads to the introduction of the Canny method for interval-valued images. Finally we include an experiment in which we compare the results of the Canny method in both scalar and interval-valued images. In that experiment the use of the latter appears to be beneficial for edge detection, over using scalar-valued images.

The remainder of this work is organized as follows. In Section 2 we analyze the measurement error in the representation of digital images. Then, in Section 3 we present the adaptation of the Canny method for interval-valued images, which performance is analyzed in Section 4. A brief discussion is included in Section 5 .

\section{Images and ambiguity representation}

\subsection{Image representation}

In this work we consider images to be matrices of $M$ rows and $N$ columns, so that $\Omega=\{1, \ldots, M\} \times$ $\{1, \ldots, N\}$ is the set of their positions. Given an image $I$, the value of a pixel at a certain position $p \in \Omega$, is referred to as $I(p)$ (e.g. may an image $I$ be grayscale, then $I(p) \in[0,1])$. Moreover, we denote as $w(p) \subset \Omega$ to the set of positions in a $3 \times 3$ neighbourhood centered at $p$, including itself. Unless $p$ belongs to the limits of the image, we have $|w(p)|=9$. 


\subsection{Images and inherent ambiguity}

Although relevant exceptions hold, digital images are the result of the discretization of the real world. That is, discrete, sampled version of continuous facts, e.g. lightning (conventional photography) or echoes to radio signals (SAR imagery). There are different sources of uncertainty to be considered when performing image processing tasks. Most of such sources are contextual, in the sense that they could be present (or not) in an image, depending on the situation the image was registered at. Evident examples of those sources of contamination are noise, distorting illumination or shading. However, there is also a source uncertainty inherent to the very nature of digital images: the measurement error. The image discretizes the reality in two different facets, spatial and tonal, each of them producing a measurement error:

- Spatial error: Surfaces and objects are continuous in reality, so that their limits are complicated to define in a precise manner. Moreover, the limits might not coincide with those in the pixel grid in the generation of the digital image. Hence, due to the way the information is stored, the digital representation might be mislocating an object by 1 position in any direction.

- Tonal error: Pixel values are taken from a finite number of tones. There are usually $2^{8}$ tones in a grayscale image and $2^{24}$ in a RGB one but, even using a larger amount of bits per pixel, there is always a limit in the tonal precision. Hence, the measure error associated to the tone of the pixel is as much as \pm 1 tone.

Both of the errors above stem from the imprecision of the discrete measure used in each dimension (spatial and tonal, respectively). Clearly, a scalar representation of pixel brightness is not sufficient for representing both errors (indeed not even one of them). Having this in mind, we propose an interval-valued representation of the image brightness, which is able to account for both errors in an explicit manner. The construction is straightly driven from the above analysis of the measurement error.

Let $I$ be a grayscale image. The interval-valued image $\breve{I}$ generated from $I$ is given by

$$
\begin{aligned}
\breve{I}(p)=[\max (0, & \left.\min _{p^{\prime} \in w(p)} I\left(p^{\prime}\right)-1\right), \\
& \left.\min \left(255, \max _{p^{\prime} \in w(p)} I\left(p^{\prime}\right)+1\right)\right] .
\end{aligned}
$$

In the construction in Eq. (1) we assign to each position in the image an interval encompassing all of the brightness values in a $3 \times 3$ neighbourhood (assuming the spatial error), modified by \pm 1 tone (because of the tonal error) [9]. Note that the interpretation of the interval is twofold. If considering

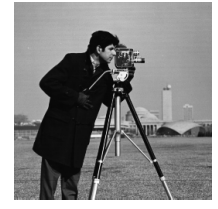

(a) Original Image
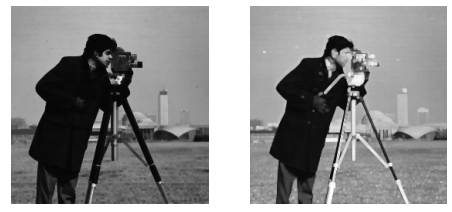

(b) IV image
Figure 1: The Cameraman image and the lower and upper bounds of its interval-valued representation.

that the pixel has one true value, it encompasses the potential values of such. Alternatively, if understanding that the real value of a pixel cannot be scalar, i.e. there is no single value than can represent the tone in that area of the image, the interval encompasses (or represents) such range of tones. The images generated from a grayscale image as in Eq. (1) are referred to as Interval-Valued Images (IV Images).

Figure 1 displays the Cameraman image, together with the upper and lower bound of its intervalvalued representation. For obvious reasons, the upper and lower bounds are very similar to dilated and eroded versions of the image [10], respectively. Also, the inverval-valued representation is similar to that produced with upper-lower constructors, as presented in [7], when using certain combination of parameters. However, the semantics of the information, as well as the use of the interval-valued information, are completely different.

The construction of IV images is robust against some types of noise (as Gaussian one), but also quite sensitive to some other kinds of contamination (as impulsive or salt-and-pepper). Dealing with external sources of contamination is not the main goal of IV images, since they only intend to capture factors intrinsically linked to the image representation.

The IV images provide a realistic interpretation of the image, since the measurement error is embedded in the original data. However, there are very few procedures in the image processing field able to deal with such a representation of the images. We devote the rest of this paper to study discrete differentiation methods, as well as to generalize the Canny method for edge detection on IV images.

\subsection{Differentiation of interval-valued signals}

The gradient of a signal is the $n$-dimensional extension of the concept of derivative. It is mathematically modelled as the vector containing the first order partial derivative in $n$ orthogonal directions. In this sense, being $S: \mathbb{R}^{n} \rightarrow \mathbb{R}$ any signal, its gradient at some position $\left(i_{1}, \ldots, i_{n}\right) \in \mathbb{R}^{n}$ is given by

$$
\nabla S\left(i_{1}, \ldots, i_{n}\right)=\left(\frac{\partial S}{\partial x_{1}}, \ldots, \frac{\partial S}{\partial x_{n}}\right)
$$

with $\left(x_{1}, \ldots, x_{n}\right)$ representing a base of vectors in $\mathbb{R}^{n}$ 
As the differentiation of discrete signals is an illposed problem, image processing algorithms typically perform directional measurements of the variation of the signal change. This is done by convolving the discrete signal with specialized filters. The study of such operators is vast and detailed [11, 12, 13], but has often rendered into the generation of antisymmetric convolution filters [14].

To the best of our knowledge, no study has been performed on the differentiation of interval-valued signals. However, from the very definition of differentiation, there is no evident problem in exporting the concept from a scalar to a interval-valued setup. When it comes to discrete signals, there is also no problem in extending the concept of discrete convolution to an interval-valued setup, as we will see in the upcoming section.

\section{The Canny method and its application to interval-valued images}

\subsection{The Canny method}

In 1983 Canny presented a master thesis [15] which was further condensed into a very significant paper [16]. The work by Canny presented several ideas, each of them receiving different attention in subsequent research. Following the trends in the literature, Canny studied the characterization of the gradients of the image as a useful representation to discriminate edges. Gradient-based edge detection methods require, at least, three steps $[17,18]$ : regularization of the image, characterization of the gradients and discrimination of those pixels for which the gradient indicates the presence of a significant boundary. The attention received by each of the three steps is very different. While regularization, either context-aware or context-unaware, has been common subject of study [19, 20], differentiation has been seldom analyzed $[21,22]$, and edge binarization is barely absent from the literature. Canny studied all three steps in the boundary detection process. However, the most acclaimed novelty in his work is the definition of three criteria the gradient extraction operators should satisfy in the context of edge detection, and derived an optimal filter for such criteria. This filter happened to be very similar to the negation of the first derivative of a Gaussian filter. As a consequence, the first order Gaussian kernels are known as Canny operators, despite Canny himself noted that the first derivative of Gaussian operator [..] should not be taken as the final word in edge detection filters, even with respect to the criteria we have used [16].

Typically, an edge detection procedure is referred to as the Canny method when it involves, sequentially, (a) Gaussian regularization of the image, (b) Gradient extraction using two orthogonal first order Gaussian kernels (see Fig. 2), (c) Non-maxima suppression for edge thinning [23] and (d) hysteresis for binarization. It should be noted that some

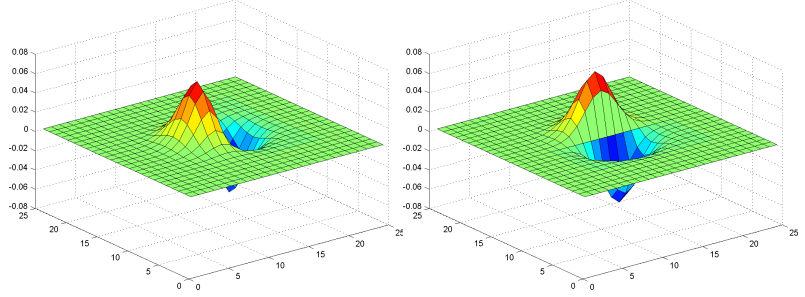

(a) Filters with $\sigma=2.0$

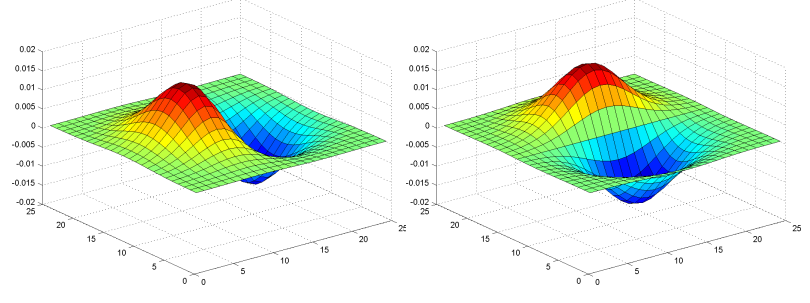

(b) Filters with $\sigma=4.0$

Figure 2: Examples of Canny differentiation operators for gradient characterization, i.e. first partial derivatives of isotropic Gaussian kernels with different $\sigma$ in two orthonormal directions.

additional procedures or techniques are needed to properly produce a binary edge image, e.g. the determination of the standard deviation of the Gaussian filters or that of the thresholds used for the hysteresis. Moreover, it is also relevant the fact that Canny proposed many other variations of this standard procedure, including anisotropic Gaussian kernels for a better adaptation to $2 \mathrm{D}$ boundaries or the fusion of gradients obtained at different scales.

Figure 3 displays an schematic representation of the application of the Canny method on the Cameraman image. First, the image is regularized using Gaussian filters, with well-known signal preservation properties [24]. Then, the gradient at a given position $p$ is constructed as the concatenation of the partial derivatives of the image, computed by convolving the image with the first derivative of a Gaussian kernel in horizontal and vertical directions. That is, $\nabla I(p)=\left(I^{x}(p), I^{y}(p)\right)$, with $I^{x}(p)=\left(I * G_{\sigma}^{x}\right)(p), I^{y}(p)=\left(I * G_{\sigma}^{y}\right)(p)$, where $G_{\sigma}^{x}$ and $G_{\sigma}^{y}$ represent the partial differentiation of a Gaussian kernel of standard deviation $\sigma$ in the horizontal and vertical axis, respectively. Once $\nabla I(p)$ is computed, many different techniques become applicable to the creation of a binary representation of the boundaries. In this case, the gradient magnitudes have been used to generate an intermediate representation of the edges, in the shape of a fuzzy set. In order to do so, we have selected the normalized Euclidean norm $\|\nabla I(p)\|=$ $\frac{1}{\sqrt{2}} \sqrt{\left(I^{x}(x, y)\right)^{2}+\left(I^{y}(x, y)\right)^{2}}$ (see $[4,25]$ for deeper discussions on other options for combining the gradient components). To finish, the conversion into binary edges has been carried out using the NonMaximum Suppression (NMS) [23] and hysteresis, combined with the double bowstring technique for thresholding $[26,27]$. 


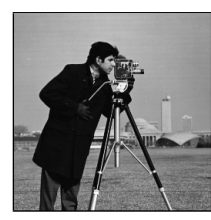

Original image

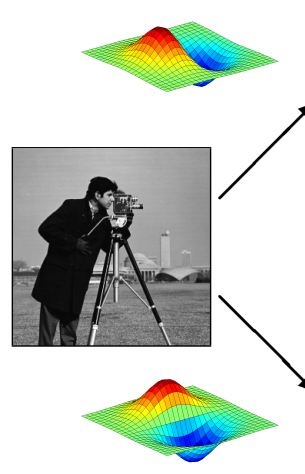

Smooth image
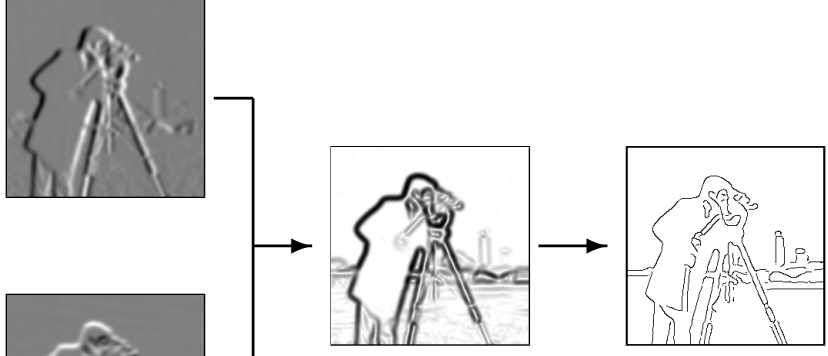

Fuzzy edges

Binary edges

Figure 3: Schematic representation of the Canny edge detection method.

\subsection{Application to interval-valued images}

The introduction of IV images poses interesting challenges to the application of the Canny method, due to the interval-valued of the signal to be differentiated. Initially, we could think of applying the detector individually on the upper and lower bounds of the intervals, as they are themselves scalar images. However, this is equivalent to considering both bounds of the interval as independent, what partially collides with the main idea behind IV images. Instead, we propose to replace the arithmetic operators in the computational model proposed by Canny to replace scalar operations by interval ones.

According to the Canny method, the partial derivative in the horizontal axis, $I^{x}$, can be computed as $I * G_{\sigma}^{x}$. For a given $p \in \Omega, p=(x, y)$, we have

$$
I^{x}(x, y)=\int_{x^{\prime}=-\infty}^{\infty} \int_{y^{\prime}=-\infty}^{\infty} I\left(x+x^{\prime}, y+y^{\prime}\right) \cdot G_{\sigma}^{x}\left(x^{\prime}, y^{\prime}\right),
$$

what becomes, in a discrete environment,

$$
I^{x}(x, y)=\sum_{i=-\infty}^{\infty} \sum_{j=-\infty}^{\infty} I(x+i, y+j) \cdot G_{\sigma}^{x}(i, j) .
$$

Since the tails of a Gaussian distribution tend to zero, we can safely restrict the support of the Gaussian filter to some $\psi=2 k+1$. In such case, we have

$$
I^{x}(x, y)=\sum_{i=-k}^{k} \sum_{j=-k}^{k} I(x+i, y+j) \cdot G_{\sigma}^{x}(i, j) .
$$

The formulation in Eq. (2) includes interval sums, differences and scalar-to-interval products, all of them well defined in interval arithmetic [28]:

- Sum of intervals: $[\underline{a}, \bar{a}]+[\underline{b}, \bar{b}]=[\underline{a}+\underline{b}, \bar{a}+\bar{b}]$;

- Difference of intervals: $[\underline{a}, \bar{a}]-[\underline{b}, \bar{b}]=[\underline{a}-\bar{b}, \bar{a}-$ b];

- Product of a positive scalar $s$ and an interval: $s \cdot[\underline{a}, \bar{a}]=[s \cdot \underline{a}, s \cdot \bar{a}]$.

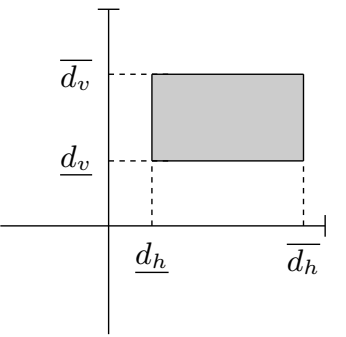

(a) Area-based interpretation

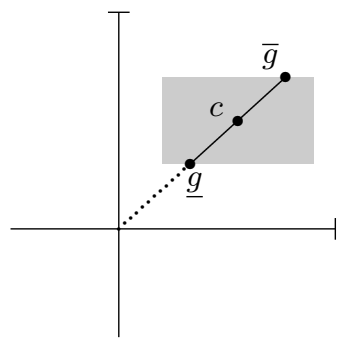

(b) Segment-based interpretation
Figure 4: Visual representation of the area gradient and its conversion to a segment. The area is arbitrarily assumed to be in the first quadrant, although analogous in any other one.

We propose to adapt the Canny method by replacing the scalar operators by interval ones. In this way, both partial derivatives of the IV image are expressed as intervals. As a consequence, the gradient estimated at the position $p$ is no longer a vector in $\mathbb{R}^{2}$, but an area instead, as illustrated in Fig. 4(a). This area can be seen as a projection of the uncertainty about the intensity of the pixels in the image (and the object boundaries). Since the initial data is unreliable, the uncertainty is propagated when measuring local features (in this case, the gradient). Following this interpretation of the image brightness, we understand that the gradient is located somewhere in the gray area in Fig. 4(a), while its exact position remains unknown.

The area-based interpretation of the gradient induces computational difficulties, more so considering our goal is to obtain a binary edge image. In this work we propose a geometric interpretation of the area in Fig. 4(a), which aims at converting the gradient area into a segment. In order to do so, we first calculate the center of gravity of the area,

$$
c(p)=\left[\frac{I^{x}(p)+\overline{I^{x}(p)}}{2}, \frac{I^{y}(p)+\overline{I^{y}(p)}}{2}\right]
$$

We take the segment created from the intersection of the area and the line passing through both 


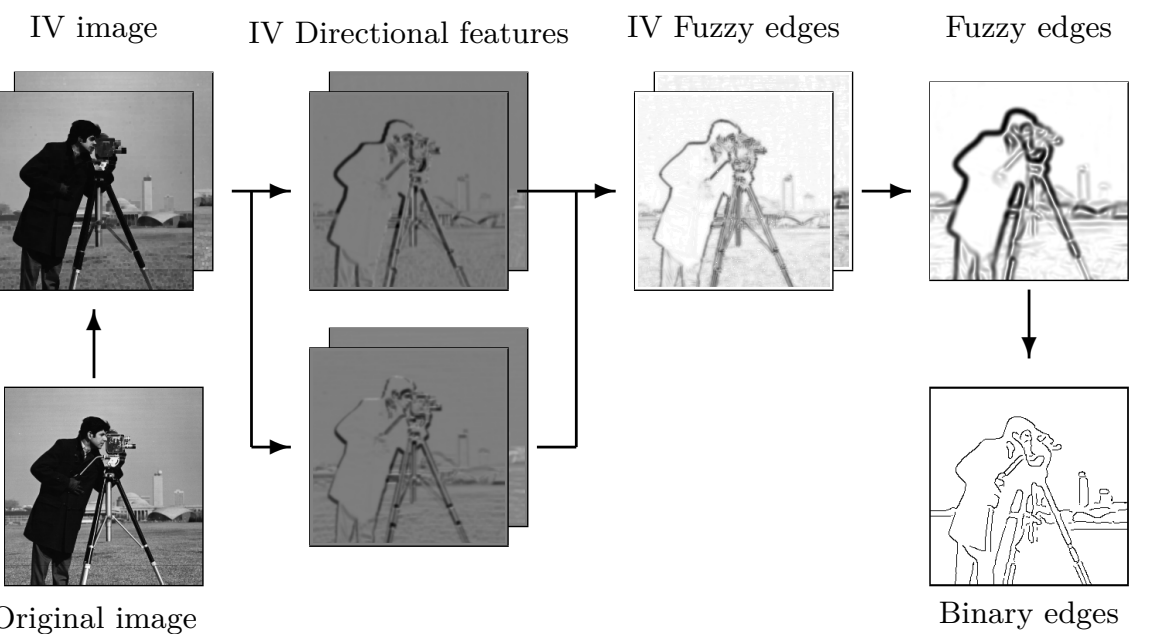

Figure 5: Visual schema of the IV Canny edge detection method

the origin $(0,0)$ and $c(p)$. From such segment, only the part belonging to the quadrant to which $c(p)$ belongs is retained. Note that, in case $c(p)$ coincides with the origin, we have a null gradient at position $p$. In Fig. 4(b), this segment is defined by $\underline{g}$ (the closest point to the origin) and $\bar{g}$ (the one furthest away).

In order to follow the Canny method as closely as possible, we have to generate a scalar representation of the edginess (computationally equivalent to a FS [29]). However, in our case, instead of creating directly a classical FS, we first use an IVFS, which we denote $G$. The interval-valued membership degree is expressed by $G(p)=[\|g\|,\|\bar{g}\|]$, where $\|\cdot\|$ stands for the normalized Euclidean norm, as before.

Before using the classical procedures for binarization, the IVFS representation of the edges is turned into a FS. To do so, we use the $K_{\alpha}$ operators, defined as $K_{\alpha}([\underline{a}, \bar{a}])=(\underline{a}+\alpha(\bar{a}-\underline{a}))$. In this way, $\alpha \in[0,1]$ represents the optimism with respect to the presence of edges. Once the edges are modeled in the shape of a FS, the processing is as it was in Fig. 3. We first use NMS for thinning the edges $^{1}$, then hysteresis, combined with the double bowstring technique for thresholding.

This method for edge detection, as illustrated in Fig. 5, is called the Interval-Valued Canny (IV Canny) method.

Figure 6 includes some results of the IV Canny method on the Cameraman image in Fig. 1(a). First, we have regularized the image with a Gaussian filter with $\sigma=1.80$. At the moment of creating a FS representation of the edges we have experimented with different values of $\alpha$. Note that the upper and lower bounds of the IVFS are the FSs generated with $\alpha=0.0$ and $\alpha=1.0$, respectively. In this figure it is noticeable that increasing optimism with respect to the appearance of boundaries, leading to greater values of $\alpha$, comes coupled to a

\footnotetext{
${ }^{1}$ The orientation of the gradient at each pixel is taken from the incidence of the segment $(g, \bar{g})$ in the origin.
}

larger number of boundary pixels. As an example, we can observe how the background buildings take shape as $\alpha$ increases. However, it remains unclear whether increasing $\alpha$ truly produce better results, since they also induce false positives (see, e.g. the grass region in the image in Fig. 6).

\section{Experimental results}

\subsection{Aim of the experiment}

The aim of this experiment is to check the effect of using IV images for edge detection. In this way, we attempt to compare the results obtained by the original Canny method on scalar images with that on interval-valued ones.

\subsection{Experimental dataset}

In this experiment we have used the test and validation subset of the Berkeley Segmentation Dataset (BSDS500) [30]. These datasets contain 200 and 100 images, respectively, together with hand-made solutions, and were published as an extension (and superset) of the original BSDS [31].

\subsection{Quantification of the results}

The evaluation of edge detection results has been an open debate for a long time [32]. In this work we approach the problem by assuming that edge detection can be seen as a binary classification problem et al. [33], and consequently can be evaluated in terms of success and fallout. We compare the output by an edge detection method (i.e. candidate edge image) with that generated by a human, which we take as ground truth. Each pixel in the candidate edge image is classified as true positive (TP), true negative $(\mathrm{TN})$, false positive $(\mathrm{FP})$ or false negative $(\mathrm{FN})$.

The classification of each edge pixel is not straightforward. There are some considerations to be taken into account, due to the special conditions 


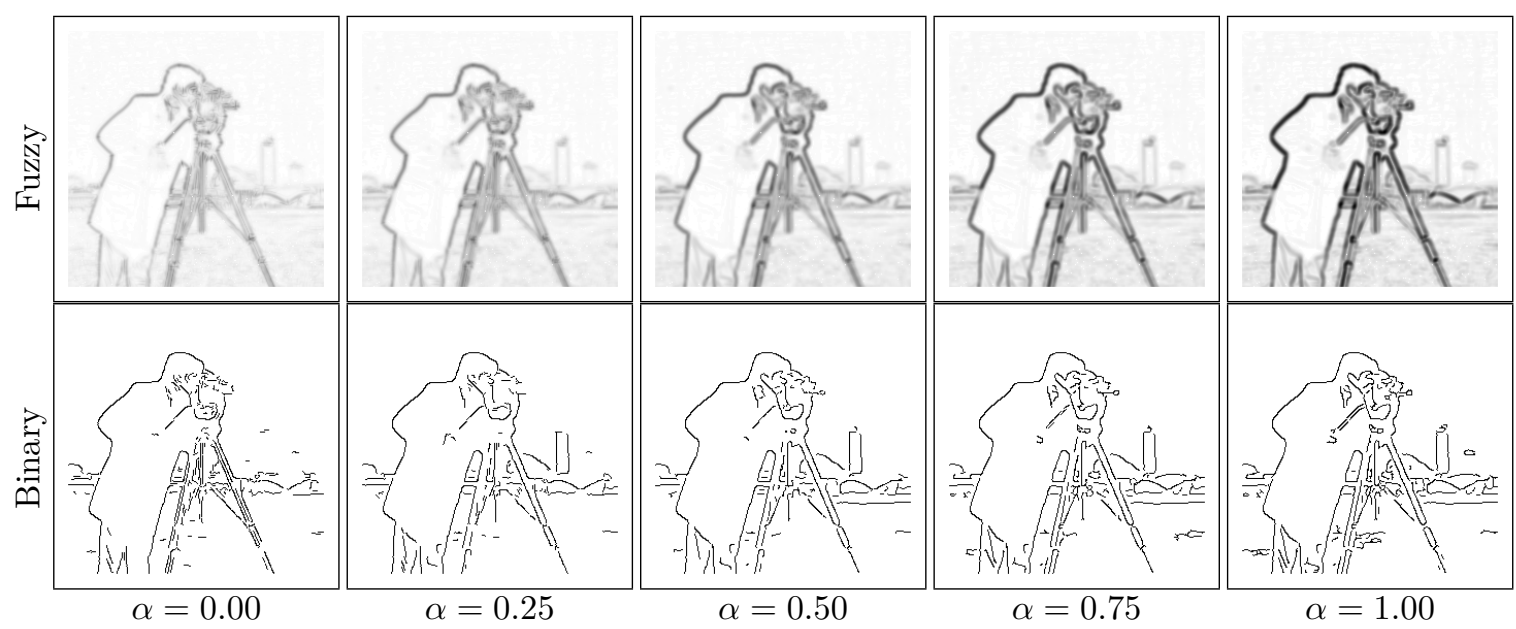

Figure 6: Fuzzy and binary edge images obtained using different $\alpha$ values for the $K_{\alpha}$ operator in the conversion of the IVFSs into FSs.

of the edge detection problem and the interpretation of the edge information. More specifically, we have to bear in mind that an edge image includes some spatial information embedded in the edges. An edge displaced from its true position should not be penalized as much as if it was completely missing. In order to solve this problem, we use a one-to-one pixel matching algorithm to map the edge pixels in the candidate edge image and the ground truth one. This matching allows some spatial tolerance (in our case, as much as $1 \%$ of the diagonal of the image), so that an edge pixel can be slightly displaced from its true position, yet being considered as correctly classified. In order to do the pixel-to-pixel matching we use the Cost Scaling Assignment algorithm by Goldberg [34].

From the confusion matrix we calculate the precision (PREC) and recall (REC) evaluations, defined as

$$
\mathrm{PREC}=\frac{\mathrm{TP}}{\mathrm{TP}+\mathrm{FP}} \text { and } \mathrm{REC}=\frac{\mathrm{TP}}{\mathrm{TP}+\mathrm{FN}} .
$$

These measures are preferred over other alternatives derived from ROC curves [35] because PREC and REC have good stability properties when the size of the images varies [30]. Moreover, they avoid involving $\mathrm{TN}$, which is much larger than the other elements in the confusion matrix, and hence distorts the results. PREC and REC evaluate specific aspects of the problem. In order to compare the performance of a method we need some scalar evaluation of the overall quality of an edge image. We use the measure $\mathrm{F}_{\alpha}$, defined as

$$
\mathrm{F}_{\alpha}=\frac{\mathrm{PREC} \cdot \mathrm{REC}}{\alpha \mathrm{PREC}+(1-\alpha) \mathrm{REC}},
$$

where $\alpha$ is a value modulating the relative impact of the PREC and REC values. Overall, we evaluate the accuracy (using PREC), the fall-out (using REC) and the overall quality (using $\mathrm{F}_{\alpha}$ ). We adhere to the commonly used $\mathrm{F}_{0.5}$ (note that $\mathrm{F}_{0.5}$ is the harmonic mean of PREC and REC).
The BSDS500 contains several ground truth images the candidate image has to be compared to. In this work, for each ground truth image we keep the triplet for which $\mathrm{F}_{0.5}$ is maximal. That is, we compare the candidate image with each of its ground truth images. Then, the triplet (PREC,REC, $\mathrm{F}_{0.5}$ ) having the greatest $\mathrm{F}_{0.5}$ is considered as the evaluation of the detector for that candidate image.

\subsection{Experimental setting}

We compare the Canny and IV Canny method on two sets of the BSDS500. For both methods, the Gaussian smoothing prior to gradient characterization is performed with $\sigma=1.0$, while the gradient characterization operators take $\sigma=2.0$. As for the binarization, we use NMS, as proposed by Rosenfeld and Kak [36], in combination with hysteresis [16], whose thresholds are determined using the doublebowstring technique by Liu et al. $[26]^{2}$.

\subsection{Results}

The results obtained at each of the two datasets in the BSDS can be observed in Fig. 7 (each column corresponding to one dataset). Each side of the figure displays the results on one of th datasets in the BSDS.

The first thing to be observed from the plots is the fact that the behaviour of the Canny method on IV images is similar for both datasets, especially regarding the the evolution of the performance of the IV Canny method with respect to $\alpha$. This indicates that the specificities of the images at each of the sets does not introduce significant differences. Another early fact that can be observed from Fig. 7 is that the performance of the IV Canny method is not smooth with respect to changes in $\alpha$. That is, small variations in $\alpha$ might induce not-so-small

\footnotetext{
${ }^{2}$ Note that this procedure is, under normal regularity conditions of the histogram, the result of two consecutive applications of the Rosin method [27].
} 
variations in the performance of the method. This is mostly due to the binarization algorithm, and the unavoidable instabilities such a process suffers.

We find clear biases in the quantitative results with respect to the value of $\alpha$. As for the precision, we observe a sudden increase for low values of $\alpha$, which is moderated for $\alpha>0.2$. In fact, the variations for $\alpha>0.2$ are minimal, and mostly due to the abovementioned discretization matters. Regarding recall, we find a slow decay with respect to the increase of $\alpha$. Overall, the optimal setting, in terms of $\mathrm{F}_{0.5}$, seems to be around the end of the fast increase of the precision, i.e. for $\alpha \approx 0.3$. Note that, in any case, the vertical axes of Fig. 7 do not cover the whole range $[0,1]$, and so the differences in performance are not drastic. As for the comparison with the Canny method, we find the use of IV images rather promising. In fact, it seems to be beneficial except for configurations with very low values of $\alpha$. Still, a more detailed comparison, as well as a re-evaluation of the remaining phases or parameters the algorithm, is needed in order to confirm this conclusion.

\section{Conclusions}

We have analyzed the role of the measurement error in digital images, proposing an interval-valued representation of the image to overcome it. With this representation we manage to model a type of error that, because of the nature of digital imagery, is embedded in the initial data. However, turning the imagery data to an interval-valued setup poses some challenges. In this work we have adapted the Canny method to Interval-Valued (IV) images, by analyzing the applicability of interval arithmetic to signal convolution. Finally, we present a quantitative comparison with the (classical) Canny method. From the results in that comparison we can infer that the performance of the Canny method can be improved by using IV images for modelling the measurement error, despite a deeper insight is needed to confirm these preliminary results.

\section{References}

[1] L. A. Zadeh, Soft computing and fuzzy logic, IEEE Software 11 (6) (1994) 48-56.

[2] F. Jacquey, F. Comby, O. Strauss, Fuzzy edge detection for omnidirectional images, Fuzzy Sets and Systems 159 (15) (2008) 1991-2010.

[3] S. K. Pal, R. A. King, On edge detection of X-Ray images using fuzzy sets, IEEE Trans. on Pattern Analysis and Machine Intelligence 5 (1) (1983) 69-77.

[4] T. Law, H. Itoh, H. Seki, Image filtering, edge detection, and edge tracing using fuzzy reasoning, IEEE Trans. on Pattern Analysis and Machine Intelligence 18 (5) (1996) 481-491.
[5] J.-A. Jiang, C.-L. Chuang, Y.-L. Lu, C.S. Fahn, Mathematical-morphology-based edge detectors for detection of thin edges in lowcontrast regions, IET Image Processing 1 (3) (2007) 269-277.

[6] S. Morillas, V. Gregori, A. Hervas, Fuzzy peer groups for reducing mixed Gaussian-impulse noise from color images, IEEE Trans. on Image Processing 18 (7) (2009) 1452-1466.

[7] E. Barrenechea, H. Bustince, B. De Baets, C. Lopez-Molina, Construction of intervalvalued fuzzy relations with application to the generation of fuzzy edge images, IEEE Trans. on Fuzzy Systems 19 (5) (2011) 819-830.

[8] P. Melin, C. I. Gonzalez, J. R. Castro, O. Mendoza, O. Castillo, Edge-detection method for image processing based on generalized type-2 fuzzy logic, IEE Trans. on Fuzzy Systems 22 (6) (2014) 1515-1525.

[9] C. Lopez-Molina, B. De Baets, B. E., B. H., Edge detection on interval-valued images, in: Advances in Intelligent and Soft Computing, Vol. 107, Springer Berlin, 2011, pp. 325-337.

[10] J. Serra, Introduction to mathematical morphology, Computer Vision, Graphics, and Image Processing 35 (3) (1986) 283-305.

[11] J. M. S. Prewitt, Object enhancement and extraction, Picture Processing and Psychopictorics, Academic Press, 1970, pp. 75-149.

[12] T. Peli, D. Malah, A study of edge detection algorithms, Computer Graphics and Image Processing 20 (1) (1982) 1-21.

[13] G. Papari, N. Petkov, Edge and line oriented contour detection: State of the art, Image and Vision Computing 29 (2-3) (2011) 79-103.

[14] N. Madrid, C. Lopez-Molina, B. De Baets, Generalized antisymmetric fiters for edge detection, in: Proc. of the Conf. of Soft Computing and Pattern Recognition, 2013.

[15] J. Canny, Finding edges and lines in images, Tech. rep., Massachussets Institute of Technology (1983).

[16] J. Canny, A computational approach to edge detection, IEEE Trans. on Pattern Analysis and Machine Intelligence 8 (6) (1986) 679-698.

[17] D. Marr, E. Hildreth, Theory of edge detection, Proceedings of the Royal Society of London 207 (1167) (1980) 187-217.

[18] V. Torre, T. Poggio, Differential operators for edge detection, Tech. rep., Massachusetts Institute of Technology (1983).

[19] T. Lindeberg, Scale-space theory in computer vision, Ph.D. thesis, KTH (Royal Institute of Technology) (1994).

[20] J. Weickert, Anisotropic Diffusion in Image Processing, ECMI Series, Teubner-Verlag, 1998.

[21] W. McIlhagga, The Canny edge detector revisited, International Journal of Computer Vision 91 (2011) 251-261. 

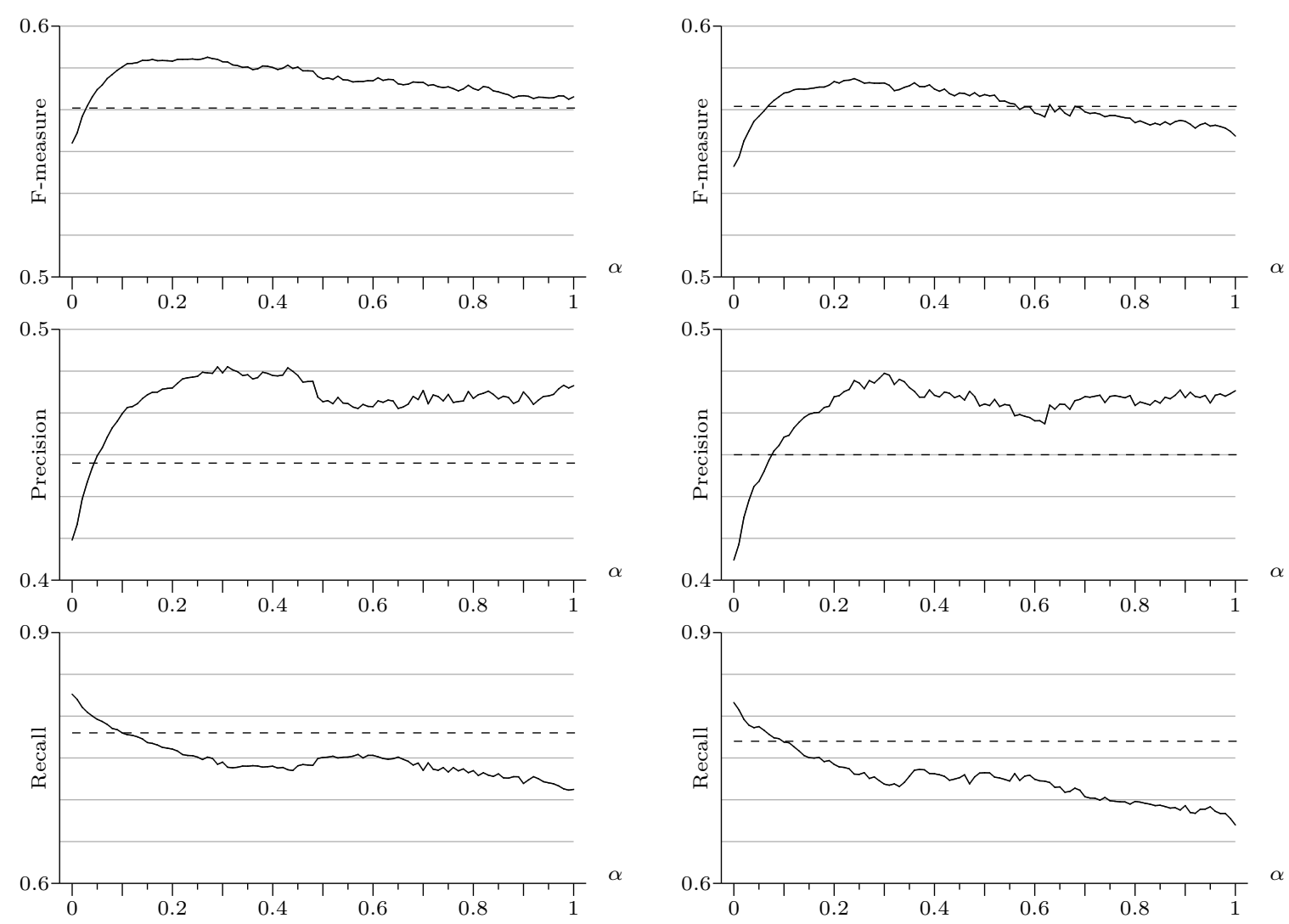

(a) Test set

(b) Validation set

Figure 7: Results by the Canny (dashed line) and IV Canny (solid line) methods on the test and validation sets of the BSDS500.

[22] P.-L. Shui, W.-C. Zhang, Noise-robust edge detector combining isotropic and anisotropic Gaussian kernels, Pattern Recognition 45 (2) (2012) 806-820.

[23] A. Rosenfeld, M. Thurston, Edge and curve detection for visual scene anaiysis, IEEE Trans. on Computers 20 (5) (1971) 562-569.

[24] T. Lindeberg, Edge detection and ridge detection with automatic scale selection, International Journal of Computer Vision 30 (2) (1998) 117-156.

[25] J. Bezdek, R. Chandrasekhar, Y. Attikouzel, A geometric approach to edge detection, IEEE Trans. on Fuzzy Systems 6 (1) (1998) 52-75.

[26] X. Liu, Y. Yu, B. Liu, Z. Li, Bowstring-based dual-threshold computation method for adaptive Canny edge detector, in: Proc. of the International Conf. of Image and Vision Computing New Zealand, 2013, pp. 13-18.

[27] P. L. Rosin, Unimodal thresholding, Pattern Recognition 34 (11) (2001) 2083-2096.

[28] R. Moore, Interval Analysis, Prentince-Hall, 1996.

[29] C. Lopez-Molina, J. Fernandez, A. Jurio, M. Galar, M. Pagola, B. De Baets, On the use of quasi-arithmetic means for the generation of edge detection blending functions, in: Proc. of the IEEE International Conf. on Fuzzy Systems, 2010, pp. 2462-2469.
[30] P. Arbelaez, M. Maire, C. Fowlkes, J. Malik, Contour detection and hierarchical image segmentation, IEEE Trans. on Pattern Analysis and Machine Intelligence 33 (2011) 898-916.

[31] D. Martin, C. Fowlkes, D. Tal, J. Malik, A database of human segmented natural images and its application to evaluating segmentation algorithms and measuring ecological statistics, in: Proc. of the IEEE International Conf. on Computer Vision, Vol. 2, 2001, pp. 416-423.

[32] C. Lopez-Molina, B. De Baets, H. Bustince, Quantitative error measures for edge detection, Pattern Recognition 46 (4) (2013) 1125-1139.

[33] D. Martin, C. Fowlkes, J. Malik, Learning to detect natural image boundaries using local brightness, color, and texture cues, IEEE Trans. on Pattern Analysis and Machine Intelligence 26 (5) (2004) 530-549.

[34] A. V. Goldberg, R. Kennedy, An efficient cost scaling algorithm for the assignment problem, Mathematical Programming 71 (1995) 153 177.

[35] W. Waegeman, B. De Baets, L. Boullart, ROC analysis in ordinal regression learning, Pattern Recognition Letters 29 (1) (2008) 1-9.

[36] L. Kitchen, A. Rosenfeld, Non-maximum suppression of gradient magnitudes makes them easier to threshold, Pattern Recognition Letters 1 (2) (1982) 93-94. 